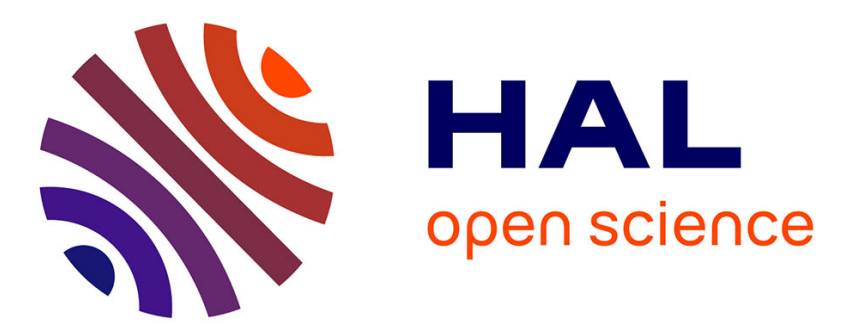

\title{
A colored hybrid Petri net-based modeling approach for performance analysis of a multi-modal transportation system
}

Karima Outafraout, El Houcine Chakir El Alaoui,, Ahmed Nait-Sidi-Moh

\section{- To cite this version:}

Karima Outafraout, El Houcine Chakir El Alaoui,", Ahmed Nait-Sidi-Moh. A colored hybrid Petri netbased modeling approach for performance analysis of a multi-modal transportation system. 2018 IEEE 14th International Conference on Automation Science and Engineering (CASE), Aug 2018, Munich, Germany. pp.1568-1573, 10.1109/COASE.2018.8560599 . hal-01993404

\author{
HAL Id: hal-01993404 \\ https://hal.science/hal-01993404
}

Submitted on 15 Feb 2019

HAL is a multi-disciplinary open access archive for the deposit and dissemination of scientific research documents, whether they are published or not. The documents may come from teaching and research institutions in France or abroad, or from public or private research centers.
L'archive ouverte pluridisciplinaire HAL, est destinée au dépôt et à la diffusion de documents scientifiques de niveau recherche, publiés ou non, émanant des établissements d'enseignement et de recherche français ou étrangers, des laboratoires publics ou privés. 


\title{
A colored hybrid Petri net-based modeling approach for performance analysis of a multi-modal transportation system
}

\author{
Karima Outafraout ${ }^{1}$, El houcine Chakir El Alaoui ${ }^{1}$ and Ahmed Nait-Sidi-Moh ${ }^{2}$
}

\begin{abstract}
This article deals with the study and analysis of a multi-modal transportation system (MTS) consisting of a bus shuttle connecting two passengers exchange stations belonging each to another transportation mode (as train lines in our case). The MTS is studied in this paper as a hybrid dynamical system (HDS). The purpose of this paper is to model, simulate and evaluate the performances of the MTS by a colored hybrid Petri nets based model (CHPNs). Owing to the developed model it is possible to analyze the system evolution over time and evaluate passengers waiting times in the two exchange stations. In addition, we show how the number of used buses on the network impact the passengers waiting times. With the aim to illustrate and validate the proposed approach, a concrete scenario is studied. Performed simulations and obtained results are reported and analyzed.
\end{abstract}

\section{INTRODUCTION}

In recent years, the growth of transportation demand and the requirements of public transport users, have made urban traffic one of the significant socio-economic issues in urban city life. At the same time, the transport networks amplification by the increasing number of vehicles, lines and stations, as well as the appearance of new concepts such as the multimodality have made public transportation and its management a major challenge to sustainable development. A MTS can be defined as the combination of two or more different modes of transport (train, bus, subway, tram, etc.) to perform the displacement of passengers (or goods) from an origin to a destination (Fig. 1). The study and the analysis of these MTSs have become one of the major preoccupations of researchers in the field of public transport to improve the quality of services provided to users. The studied MTS in this paper includes two exchange stations belonging each to a train line as illustrated by red circle in Fig. 1. The passengers exchange between these stations is ensured by bus shuttles. In this paper, we are interested by modeling, analyzing and evaluating performances of the studied MTS in terms of passengers waiting times.

Similarly to manufacturing systems and communications networks, MTSs can be seen as HDSs. The behavior of these systems is mainly characterized by two kinds of phenomena : discrete events corresponding to the arrivals and departures of the transportation means (trains, buses,...),

\footnotetext{
${ }^{1}$ Karima Outafraout and El houcine Chakir El Alaoui are with Laboratory of Electrical and Telecommunication Systems, Cadi Ayyad University, 40000 Marrakech,

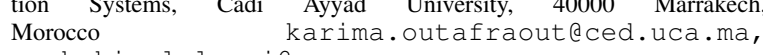
e.chakirelalaoui@uca.ma

${ }^{2}$ Ahmed Nait-Sidi-Moh is with the Laboratory of Innovative Technologies, Picardie Jules Verne University, France ahmed.nait@u-picardie.fr
}

and continuous dynamics corresponding to the movements of passengers (as continuous flows) into the exchange stations. In fact, some interactions exist between the discrete events and the continuous dynamics which influence each others behavior. For example, the vehicles arrivals and departures control the start and the end of disembarking and boarding operations of passengers. In order to analyze and represent the complex behavior of the considered MTSs, the first step is to well understand, thanks to an appropriate model, the internal functioning of these systems. Indeed, hybrid Petri nets (HPNs) have been proven to be a powerful modeling formalism for various kinds of HDSs and more precisely, the sub class CHPN allows to get a simpler and compact form of the complex structure of these systems. Based on the assets of this formalism, and in order to reach the above mentioned objectives, we developed a CHPNs based model to describe the MTS behavior. Afterwards, its properties and performances will be evaluated and analyzed.

This paper is organized as follows. In section II, we address a related work. A description of the studied MTS is given in section III. Then, the CHPNs model of the studied system is presented in section IV. An illustrative numerical example and obtained results are presented in section $\mathrm{V}$. The last section concludes the paper and gives some future perspectives.

\section{RELATED WORK}

The dynamics of a public transport systems are usually modeled as a discrete event systems. Discrete approaches are suitable for describing the operation of transportation when the number of the exchange passengers is considered within a countable set. During peak period for instance, there exists a large number of passengers into the stations. This may result the explosion of the number of reachable states. Therefore, exploiting the model may be difficult or impossible. Thus, the whole passengers can be considered as a homogeneous set of passengers and their collective movements look like continuous flows. Therefore, a continuous approach will be a good approximation of the passengers dynamics into the stations. Consequently, the MTS can be considered as a HDS because of the discrete and continuous aspects of its components. However, the behavior of these systems is often extremely complex because of the interaction of these components.

In the literature, HPNs [6] were successfully applied for modeling, simulation and performance evaluation of several HDSs as manufacturing systems [2], communication networks [5], microgrid systems [4] and they have also been 


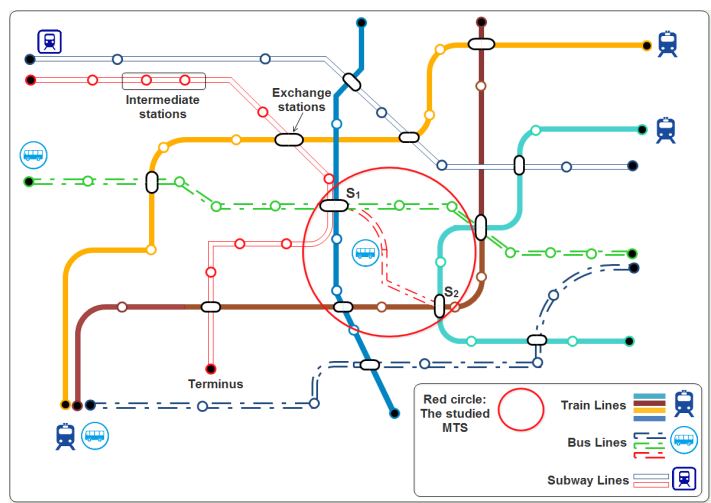

Fig. 1. A multi-modal transportation network

successfully used for studying transportation systems [1], [7], [8]. For example, the authors of [2] model and manage an inter-modal freight transport terminals loaded with goods by a first-order HPNs. The proposed approach allows taking decisions on the management of transfer speeds and the quality of resources needed for inter-modal transport units transferring in case of variation of commercial flows in the terminal. An optimization approach for congestion management of traffic signal control systems has been proposed in [1] and [7]. The authors of [8] propose a modeling approach based on CHPNs to model the urban signalized intersection in order to optimize the traffic signal control of isolated intersections.

The current paper presents an extension of our research work presented in [3]. In this paper, we are interested in determining a compromise between the number of used buses on the network and the passengers waiting times within the exchange stations (use a minimum number of buses while reducing waiting times of passengers).

\section{Description OF THE STUdied MTS}

In this paper, we are interested in studying the evolution of passengers flows transiting between two exchange stations $\left(S_{1}\right.$ and $S_{2}$ ) as illustrated in Fig. 1. The passengers exchange is ensured by shuttles. Each exchange station belongs to one or more transportation modes (train, subway, tram, etc.). Each public transportation mode makes a circuit from its departure station to its arrival station, while serving other lines of the same mode or those of other modes via exchange stations. Our approach will be illustrated on a MTS which is composed of a bus line ensuring a shuttle between $S_{1}$ and $S_{2}$ belonging each to a train line as illustrated in Fig. 1 (by red circle). Each station contains a buses depot (we suppose that each depot contains a sufficient number of buses to ensure the transportation service between $S_{1}$ and $S_{2}$ ). When the number of buses already circulating on the network is not enough or is insufficient to respect a giving passengers waiting time (defined specification and denoted Spec) the buses of station depot feed the network in order to transport waiting passengers. The number of transiting buses between the two stations is denoted $n$. Each bus is characterized by its capacity $C(C$ is the maximum number of passengers that can board a bus at the same time). We consider also that the train capacities are infinite (which means that all bus passengers can board into the trains). We suppose that the buses departure from the exchange stations is allowed only if the departure order is given after boarding of waiting passengers. Whereas, we consider that each train arriving at the exchange station leaves it after disembarking and/or boarding of passengers and that after a fixed time interval another train arrives at the station and so on.

\section{MODELING APPROACH WITH CHPNS}

In order to present the complex behaviour of our system, we have developed a CHPNs-based model whose continuous part is represented by a constant speed continuous Petri nets (CCPNs) and discrete part is represented by a timed Petri net and the tokens are identified by colors in order to preserve the information concerning each transportation means and stations. As proved in the litterature, CHPN is a powerful tool for modeling and analyzing hybrid systems. Indeed, this tool allows to obtain: (i) a graphical representation of the different parts (discrete and continuous) of the system, (ii) an easy and efficient modeling of the characteristics of MTS, (iii) and a performance evaluation. For further detail about this tool, we refer the reader to [6]. In this section, the proposed CHPNs based model is presented.

\section{A. Modeling framework}

In this paper, we assume that the bus capacity $C$ is limited and is the same for all buses. For the buses, passengers disembarking has priority over boarding (sequential approach), while for the trains passengers we consider that the disembarking and the boarding take place simultaneously (parallel approach). We consider that there is no synchronization between the two modes of transportation (trains and buses). We also consider that the passengers who are going to disembark from the trains are represented by the random natural numbers $N_{1}$ (for the first train line) and $N_{2}$ (for the second one) according to the period nature (peak or offpeak period). Let recall that "peak period" (resp. "off-peak period") the period when $N_{j}(\forall j \in\{1,2\})$ is large (resp. small) compared to the bus capacity $\mathrm{C}$. For example, we consider that during a peak period $N_{j}>2^{*} C$ while during an off-peak period $N_{j} \leq C$. Otherwise, when $C<N_{j}<2^{*} C$ we consider that the passenger amount is normal.

\section{B. CHPN model of the studied MTS}

In this section, the developed CHPN model of the studied MTS is given in Fig. 2. It's obvious that the $n$ buses have the same behavior and the operation described in $S_{1}$ (passengers exchange between trains and buses in $S_{1}$ ) is similar to those in $S_{2}$. Therefore, the proposed CHPN allows us to have a simpler and compact model of the complex behavior of the system. In the CHPN model, we find three sets of colors associated with places and transitions : 


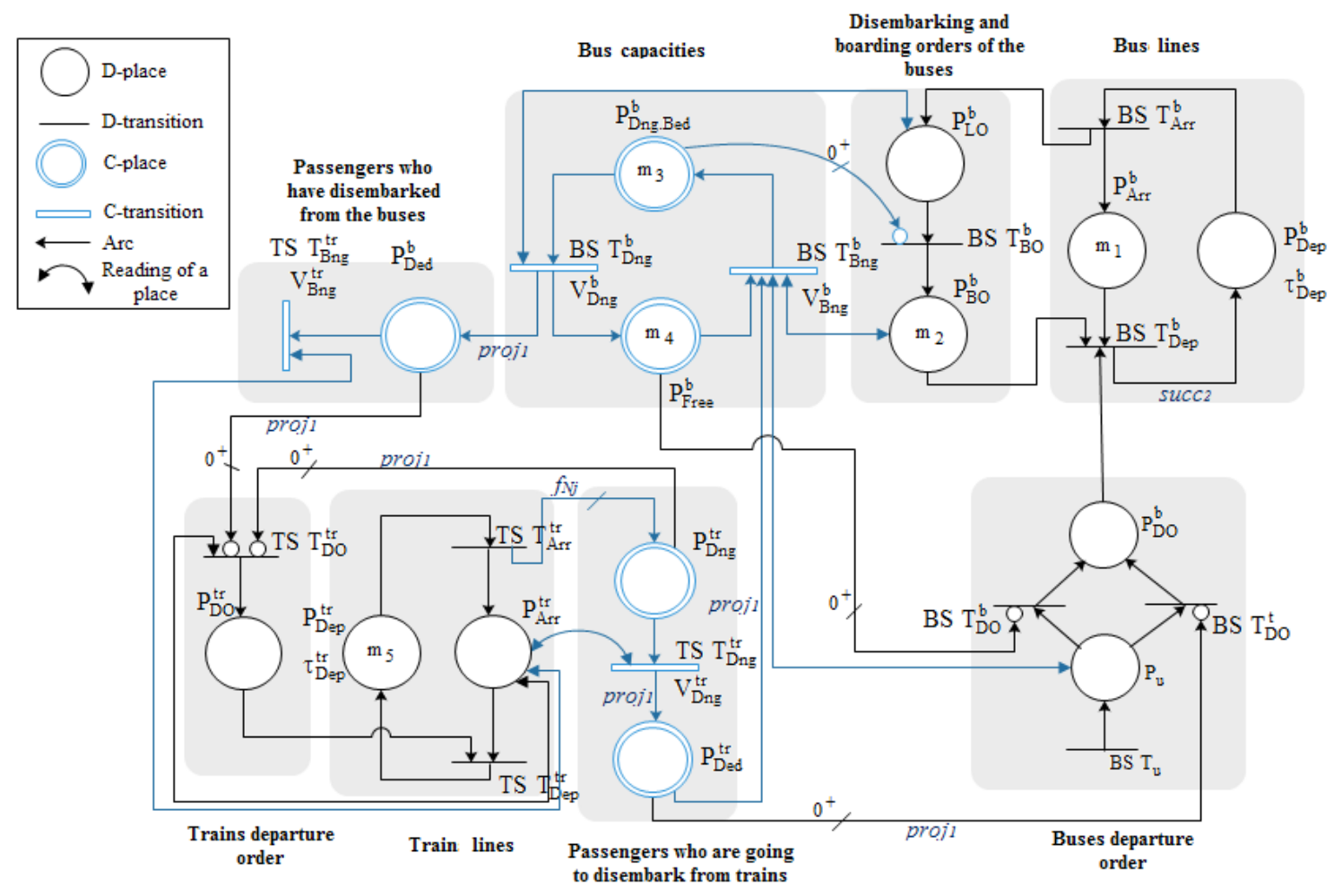

Fig. 2. Colored hybrid Petri net of the studied MTS

- $B=\left\{b_{1}, b_{2}, \ldots, b_{n}\right\}$ represents the set of colors associated with the buses and $n$ is the number of buses in the network (shuttle circuit).

- $S=\left\{s_{1}, s_{2}\right\}$ corresponds to the set of colors associated with the two exchanges stations $S_{1}$ and $S_{2}$.

- $T=\left\{t_{1}, t_{2}\right\}$ corresponds to the set of colors associated with the two trains lines, $t_{1}$ for the first train line and $t_{2}$ for the second one.

Two composed colors sets $\left(\left\langle b_{i}, s_{j}\right\rangle\right.$ and $\left\langle t_{j}, s_{j}\right\rangle$ such as $\left\langle b_{i}\right\rangle \in B,\left\langle s_{j}\right\rangle \in S$ and $\left.\left\langle t_{j}\right\rangle \in T\right)$ are used in the developed model in Fig. 2. The composed color $\left\langle b_{i}, s_{j}\right\rangle$ (resp. $\left\langle t_{j}, s_{j}\right\rangle$ ) associate to each station its buses (resp. trains).

The tables I and II describe the initial marking and the functions used in the developed model (Fig. 2). The initial marking of all places which are not indicated in table I equals to zero. Now, let us present the description of the nodes of the CHPN model (Fig. 2).

1) Vehicle arrivals and departures : the arrivals and departures of the vehicles (trains and buses) are modeled using the discrete P-timed Petri nets (discrete events). In the following, the places and transitions which represent the bus and train arrivals and departures are presented.

Lines of buses : the discrete place $P_{A r r}^{b}$ models the two exchange stations $S_{1}$ and $S_{2}$. A token $\left\langle b_{i}, s_{j}\right\rangle$ in $P_{A r r}^{b}$ means that the bus $b_{i}$ is present at the exchange station $S_{j}$. The initial marking of $P_{A r r}^{b}$ is $m_{1}$ (see table I) and it means
TABLE I

INITIAL MARKING OF THE CHPN OF FIG. 2

\begin{tabular}{cc}
\hline Place & Value \\
\hline$P_{A r r}^{b}$ & $m_{1}=\left(\bigcup_{i=1}^{m}\left\langle b_{i}, s_{1}\right\rangle, \bigcup_{i=m+1}^{n}\left\langle b_{i}, s_{2}\right\rangle\right)$ \\
$P_{B O}^{b}$ & $m_{2}=m_{1}$ \\
$P_{D n g . B e d}^{b}$ & $m_{3}=\left(\bigcup_{i=1}^{m} c_{p i 1}\left\langle b_{i}, s_{1}\right\rangle, \bigcup_{i=m+1}^{n} c_{p i 2}\left\langle b_{i}, s_{2}\right\rangle\right)$ \\
$P_{F r e e}^{b}$ & $m_{4}=\left(\bigcup_{i=1}^{m} c_{v i 1}\left\langle b_{i}, s_{1}\right\rangle, \bigcup_{i=m+1}^{n} c_{v i 2}\left\langle b_{i}, s_{2}\right\rangle\right)$ \\
$P_{\text {Dep }}^{t r}$ & $m_{5}=\left(\left\langle t_{1}, s_{1}\right\rangle,\left\langle t_{2}, s_{2}\right\rangle\right)$ \\
\hline
\end{tabular}

TABLE II

USED COLOURED FUNCTION IN CHPN OF FIG. 2

\begin{tabular}{cc}
\hline Function & Definition \\
\hline succ $_{2}$ & $\operatorname{succ}_{2}\left(\left\langle b_{i}, s_{j}\right\rangle\right)=\left\langle b_{i}, s_{j+1}\right\rangle($ modulo 2 for $j)$ \\
& $\operatorname{proj}_{1}\left(\left\langle b_{i}, s_{j}\right\rangle\right)=$ proj $_{1}\left(\left\langle t_{j}, s_{j}\right\rangle\right)=\left\langle s_{j}\right\rangle$ \\
$f_{N j}$ & $f_{N j}\left(\left\langle t_{j}, s_{j}\right\rangle\right)=N_{j}\left\langle t_{j}, s_{j}\right\rangle$ \\
\hline
\end{tabular}

that, initially, there are $m$ buses in $S_{1}$ and $(n-m)$ buses in $S_{2}(n>m)$. The buses are empty and wait for the departure order. It's necessary to note that $m$ and $n$ will be dynamics according to the period nature.

$P_{D e p}^{b}$ is a discrete timed place which represents the displacement of the bus between $S_{1}$ and $S_{2}$. A token $\left\langle b_{i}, s_{j}\right\rangle$ in $P_{D e p}^{b}$ means that the bus $b_{i}$ is in displacement towards $S_{j}$. The time $\tau_{D e p}^{b}$ associated with $P_{D e p}^{b}$ corresponds to the travel time between the two stations (which is supposed to 
be the same in the two directions).

The discrete transition $T_{A r r}^{b}$ (resp. $T_{D e p}^{b}$ ) represents the bus arrivals at (resp. departures from) $S_{1}$ or $S_{2}$. The set of colors associated with these transitions is $B S$ which means that the transitions $T_{A r r}^{b}$ and $T_{D e p}^{b}$ may be fired by any color $\left\langle b_{i}, s_{j}\right\rangle$. The function $s_{u c c_{2}}$ associated to the arc between $T_{D e p}^{b}$ and $P_{D e p}^{b}$ performs a linear transformation of the colors associated with the transition $T_{D e p}^{b}$. For example, the firing of the transition $T_{D e p}^{b}$ by any color $\left\langle b_{i}, s_{1}\right\rangle$ consists of removing $\left\langle b_{i}, s_{1}\right\rangle$ from the place $P_{A r r}^{b}$ and adding $\left\langle b_{i}, s_{2}\right\rangle$ $\left(\operatorname{succ}_{2}\left(\left\langle b_{i}, s_{1}\right\rangle\right)=\left\langle b_{i}, s_{2}\right\rangle\right)$ at $P_{D e p}^{b}$ which means that the bus $b_{i}$ left $S_{1}$ and is in displacement towards $S_{2}$. The others arcs of the model without color functions correspond to the identity $(i d)$ function (which means no color change when firing the transition).

Lines of trains : the discrete sequence $\left(T_{A r r}^{t r}, P_{A r r}^{t r}, T_{D e p}^{t r}\right.$ $\left.P_{D e p}^{t r}\right)$ models the two train lines with their arrivals $T_{A r r}^{t r}$ and departures $T_{D e p}^{t r}$ at/from $S_{1}$ (for train $t_{1}$ ) and $S_{2}$ (for train $t_{2}$ ). The set of colors associated with the transitions $T_{A r r}^{t r}$ and $T_{D e p}^{t r}$ is $T S$. A token $\left\langle t_{j}, s_{j}\right\rangle$ in $P_{A r r}^{t r}$ means that the train $t_{j}$ arrived at $S_{j}$ whereas a token in $P_{D e p}^{t r}$ means that it leaves the station. The time $\tau_{D e p}^{t r}$ denotes the interval between the train $t_{j}$ departure and the next arrival of another train at $S_{j}(j \in\{1,2\})$. Initially, we suppose that the two trains are in displacement to their corresponding stations $\left(m_{5}=\left(\left\langle t_{1}, s_{1}\right\rangle,\left\langle t_{2}, s_{2}\right\rangle\right)\right)$ (see table I).

2) Exchange of passengers flow: it's easy to see that the global capacity of a shuttle bus $b_{i}$ is the sum of the numbers of tokens of the two places $P_{D n g . B e d}^{b}$ and $P_{\text {Free }}^{b}$ associated with the same color $\left\langle b_{i}, s_{j}\right\rangle$ (Fig. 2), where :

- The continuous place $P_{D n g . B e d}^{b}$ corresponds to the number of passengers that will disembark on the exchange stations (or equivalently the boarded passengers into the buses). The marking $c_{p i j}\left\langle b_{i}, s_{j}\right\rangle$ (table I) corresponds to the number of passengers on board of the bus $b_{i}$ at $S_{j}$

- $P_{F r e e}^{b}$ is a continuous place which represents the number of empty seats into the buses expressed in number of passengers (denoted $p s g$ ). The marking $c_{v i j}\left\langle b_{i}, s_{j}\right\rangle$ (table I) represents the free capacity of the bus $b_{i}$ at $S_{j}$.

Then, it's obvious that $c_{p i j}\left\langle b_{i}, s_{j}\right\rangle+c_{v i j}\left\langle b_{i}, s_{j}\right\rangle=C$.

$T_{D n g}^{b}\left(\right.$ resp. $\left.T_{B n g}^{b}\right)$ is a continuous transition which models the passengers disembarking (resp. boarding) through the buses gates. The set of colors associated with $T_{D n g}^{b}$ (resp. $T_{B n g}^{b}$ ) is $B S$. So, the transition $T_{D n g}^{b}$ (resp. $T_{B n g}^{b}$ ) may be fired by any color $\left\langle b_{i}, s_{j}\right\rangle$ which corresponds to the passengers disembarking from the bus $b_{i}$ on $S_{j}$.

The continuous place $P_{D e d}^{b}$ models the passengers who have disembarked from the buses on the two exchange stations and are going to board into the trains.

$T_{B n g}^{t r}$ (resp. $T_{D n g}^{t r}$ ) is a continuous transition which represents the passengers boarding into (resp. disembarking from) the trains. The set of colors associated with $T_{B n g}^{t r}$ and $T_{D n g}^{t r}$ is $T S$. The firing of $T_{B n g}^{t r}$ by the color $\left\langle t_{j}, s_{j}\right\rangle$ means that the passengers board into the train $t_{j}$.

$P_{D n g}^{t r}\left(\right.$ resp. $\left.P_{D e d}^{t r}\right)$ is a continuous place which models the passengers who are going to disembark (resp. disembarked passengers) from the trains on the two stations. When the train $t_{1}$ arrives at $S_{1}$ (firing of $T_{A r r}^{t r}$ by $\left\langle t_{1}, s_{1}\right\rangle$ ) we remove one token $\left\langle t_{1}, s_{1}\right\rangle$ from $P_{D e p}^{t r}$ and adding $N_{1}\left\langle t_{1}, s_{1}\right\rangle$ tokens of the same color (by the function $f_{N j}$ ) to $P_{D n g}^{t r}$ which corresponds to the numbers of passengers who want to disembark on $S_{1}$.

3) Disembarking and boarding orders : as aforementioned, in this paper the passengers disembarking take priority over the boarding for the buses. This priority is ensured by the following discrete places and transitions :

$P_{L O}^{b}$ (resp. $P_{B O}^{b}$ ) is a discrete place which represents the disembarking (resp. boarding) order of bus passengers on the stations. The firing of the discrete transition $T_{B O}^{b}$ by $\left\langle b_{i}, s_{j}\right\rangle$ allows the boarding order on the bus $b_{i}$ at $S_{j}$. This firing is possible only if all passengers were disembarked from $b_{i}$ which corresponds to a zero test of the place $P_{D n g \text {.Bed }}^{b}$. The combination of the weight $0^{+}$with an inhibitor arc allows to perform the zero test of a continuous place. For more details about the zero test in a continuous place we refer to [6].

4) Departure orders of the buses : $P_{D O}^{b}$ is a discrete place which models the departure order of the buses from the stations. The departure order of the bus $b_{i}$ from $S_{j}$ is given (token $\left\langle b_{i}, s_{j}\right\rangle$ in $P_{D O}^{b}$ ) after both receiving the departure order (token $\left\langle b_{i}, s_{j}\right\rangle$ in $P_{u}$ ) and the bus is full or if there are no waiting passengers in $S_{j}$.

The discrete transition $T_{D O}^{b}$ is fired only if the marking associated with $\left\langle b_{i}, s_{j}\right\rangle$ in the place $P_{F r e e}^{b}$ equals to zero (which means that the bus $b_{i}$ is full) and the firing of the transition $T_{D O}^{t}$ is possible only if the marking associated with $\left\langle s_{j}\right\rangle$ in the place $P_{D e d}^{t r}$ equals to zero (which means that there is no more waiting passengers in $S_{j}$ ), it corresponds to a zero test of these places (inhibitor arcs with $0^{+}$).

5) Departure orders of the two trains : the discrete place $P_{D O}^{t r}$ models the departure order of the trains from the exchange stations. The firing of the discrete transition $T_{D O}^{t r}$ authorizes the trains departure if both all passengers are boarded into the trains and all passengers are disembarked from theses trains, which corresponds to the zero test of the two places $P_{D e d}^{b}$ and $P_{D n g}^{t r}$.

\section{Firing speeds of the continuous transitions}

Two algorithms have been proposed in the literature for defining the instantaneous speeds of continuous transitions [6]. In this study, we will use the CCPN-based (Constant speed Continuous Petri Net-based) algorithm for which the firing speed vector of the CHPN remains constant during each invariant behavior phase (i.e., time interval between two events which change the behavior of the model).

The maximal firing speeds $V_{B n g}^{b}$ and $V_{D n g}^{b}$ correspond respectively to the boarding and disembarking flows in/from the buses on the stations respectively. In the same way, $V_{B n g}^{t r}$ and $V_{D n g}^{t r}$ denote respectively the boarding and disembarking flows in/from the trains. We suppose that the boarding and disembarking speeds of all buses (resp. trains) are equals. 


\section{Simulation AND EVALUATION}

\section{A. Adopted methodology}

The simulation of a HPNs is based on the determination of its Invariant Behavior States (IBSs) using (1). An IB-state is defined such that the marking of the discrete places and the instantaneous speed vector of the continuous transitions remain constant as long as the system is in the same IB-state. Formally, this is expressed as follows :

$$
\begin{gathered}
\forall t \in I B S_{k}=\left[t_{i k} t_{f k}\right] \\
m(t)=m\left(t_{i k}\right)+W_{k} \cdot\left[\sigma\left(t-t_{i k}\right)+\int_{t_{i k}}^{t} v(u) d u\right]
\end{gathered}
$$

where, $\left[t_{i k} t_{f k}\right]$ corresponds to the time slot of the $k^{t h}$ IBS. $W_{k}$ is the incidence matrix. The vector $\sigma\left(t-t_{i k}\right)$ represents the firing number of each discrete transition between $t_{i k}$ and $\mathrm{t} \leq t_{f k}$ (the components of $\sigma$ associated with continuous transitions are null). The vector $v(u)$ symbolizes the instantaneous firing speeds associated with continuous transitions (the components of $v$ associated with discrete transitions are null). For more details we refer to [6].

\section{B. Numbers of exchange passengers}

The number $N_{j}$ of passengers who are going to disembark from the train $t_{j}$ ( $N_{j}$ is given by the colored function $f_{N j}$ associated to the arc between $T_{A r r}^{t r}$ and $P_{D n g}^{t r}$ (Fig. 2)), is a parameter which is not a priori known for each train arrival. So, for simulation purposes, we propose to randomly generate these numbers according to peak and off-peak periods. These numbers will take stochastic values comprised between two bounds $\left(C_{\text {Inf.N. }}\right.$ and $\left.C_{\text {Sup. } N_{j}}(j \in\{1,2\})\right)$ according to the following equation :

$$
N_{j}=C_{\text {Inf. } N_{j}}+\operatorname{rand}\left(C_{{\text {Sup. } N_{j}}_{j}}-C_{\text {Inf. } N_{j}}\right)
$$

where rand is a generator function of uniformly distributed random numbers such that : for all $x, 0 \leq \operatorname{rand}(x) \leq x$. As in real situation, the artificial number $N_{j}$ may be higher, less or equal to the buses capacity $C$ depending on the period.

\section{Simulation results}

To prove our methodology, we have performed some simulations using Matlab software. The simulation algorithm is based on the determination of the whole IBSs of the studied system in a finite simulation time. The tables III and IV recapitulate the required data for the simulation of the developed model.

According to the bus capacities, the train passengers $N_{j}$ $(j \in\{1,2\})$ are divided into lots of passengers. Each passengers lot is inferior or equal to the bus capacity $C$ such as all passengers of the same lot take the same bus. For example, let's take $N_{1}=70 \mathrm{psg}$. Hence, $N_{1}$ will be divided into two lots of passengers, the first one contains $50 \mathrm{psg}$ (which corresponds to the bus capacity) and the second one contains $20 \mathrm{psg}$. Two shuttle buses are then required to ensure the transport of these two passengers lots. The waiting time of a passengers lot is the difference between the departure time of the bus that will take these
TABLE III

INPUT DATA OF THE CHPN (FIG. 2)

\begin{tabular}{cc}
\hline DATA & VALUE \\
\hline Bus Capacity $(C)$ & $50 \mathrm{psg}$ \\
Bus travel time between $S_{1}$ and $S_{2}\left(\tau_{B . D e p}\right)$ & $15 \mathrm{~min}$ \\
Trains travel time $\left(\tau_{D e p}^{t r}\right)$ & $25 \mathrm{~min}$ \\
Buses disembarking and boarding flows & \\
$V_{B n g}^{b}=V_{D n g}^{b}$ & $30 \mathrm{psg} / \mathrm{min}$ \\
Trains disembarking and boarding flows & \\
$V_{B n g}^{t r}=V_{D n g}^{t r}$ & $120 \mathrm{psg} / \mathrm{min}$ \\
\hline
\end{tabular}

TABLE IV

INPUT PARAMETERS OF THE GENERATION PROCESS OF $N_{1}$ AND $N_{2}$

\begin{tabular}{ccc}
\hline Artificial number & \multicolumn{2}{c}{ Parameters of equation 2 } \\
\cline { 2 - 3 } & Off-peak period & peak period \\
\hline$N_{j}(j=1,2)$ & $C_{\text {Inf. } N_{j}}=70$ & $C_{\text {Inf. } N_{j}}=120$ \\
& $C_{\text {Sup. } N_{j}}=100$ & $C_{\text {Sup. } N_{j}}=200$ \\
\hline
\end{tabular}

passengers and the arrival time of the train that brought them at the station. As aforementioned, one of the purposes of this contribution is to determine the minimum number of buses to be used while respecting the considered specification. We fix this specification to $20 \mathrm{~min}$ for the two stations. When the train $t_{j}$ arrives at $S_{j}$ we define the number of needed buses for transporting these passengers to the other station. Then, we calculate the remaining time of the all circulating buses in the network to arrive to $S_{j}$. This time plus the passengers disembarking and boarding times must be less than or equals to $S p e c=20 \mathrm{~min}$. If the number of buses (already circulating between the two stations) that respect the specification is insufficient, we inject other buses into the network from the available buses on the station depot. This injection takes into account the buses already on the network and that respect the specification.

Studied scenario : The performed simulation for this scenario (Fig. 3, 4, 5, and 6) corresponds to an off-peak period of 1 hour followed by a peak period of 2 hour itself followed by a second off-peak period of 1 hour. During these periods, stochastic numbers $N_{1}$ and $N_{2}$ (Fig. 3) are generated using (2) with parameters of table IV.

The performed simulation in Fig. 3 corresponds to the variation of passengers numbers $N_{1}$ and $N_{2}$ who are going to disembark from the two trains on $S_{1}$ and $S_{2}$, respectively. Fig. 4 represents the waiting times of these passengers in the two stations for $n=4$ buses during peak and offpeak periods. By analyzing this figure, we remark that the specification is respected in off-peak period whereas it is not respected in peak period. The specification is respected for $70 \%$ in this case (Fig. 4). In order to increase the specification satisfaction, we inject 1 bus into the network from the stations depot (Fig. 5). By this injection we notice that the waiting time decreases compared to the Fig. 4, and the specification satisfaction reaches $90 \%$. To improve the quality of service provided to passengers to $100 \%$ during the two periods, we inject 2 buses during peak period. Indeed, to respect $100 \%$ of the specification (Fig. 6) the minimum number of buses to be used in off-peak period is 

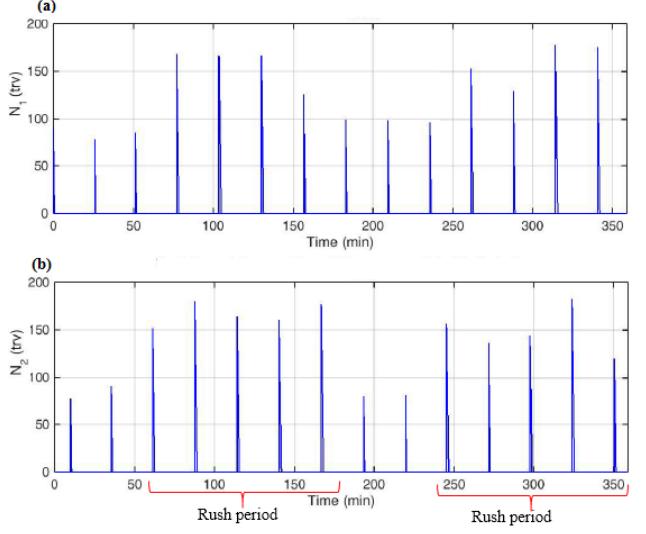

Fig. 3. Variation of the artificial numbers $N_{1}$ (a) and $N_{2}$ (b)
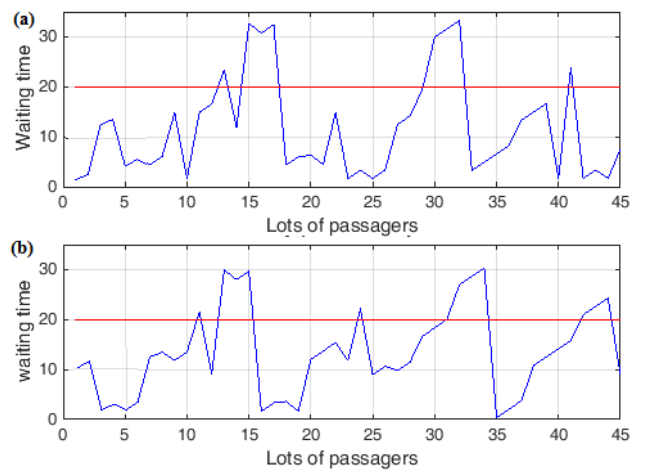

Fig. 4. Variation of the waiting time in $S_{1}$ (a) and $S_{2}$ (b) for $n=4$ buses
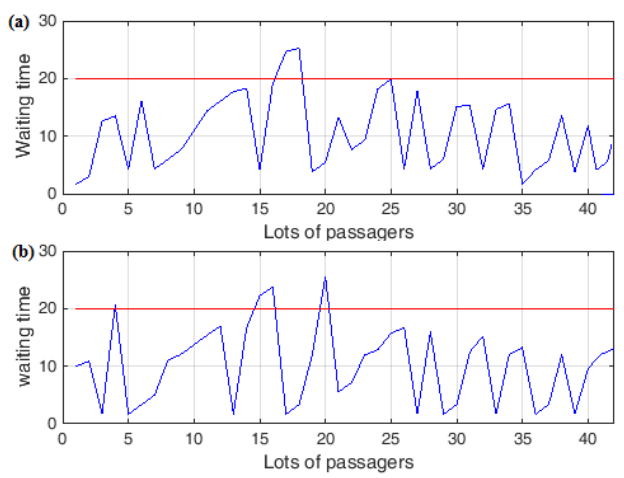

Fig. 5. Variation of the waiting time in $S_{1}$ (a) and $S_{2}$ (b) for $n=5$ buses

$n=4$ buses, while in peak period $n=6$ buses.

Consequently, the number of needed buses for exchanging the passengers between the two stations depend on the working period. Indeed, it turns out that a compromise must be found between the number of used buses on the network and the passengers waiting times on the exchange stations.
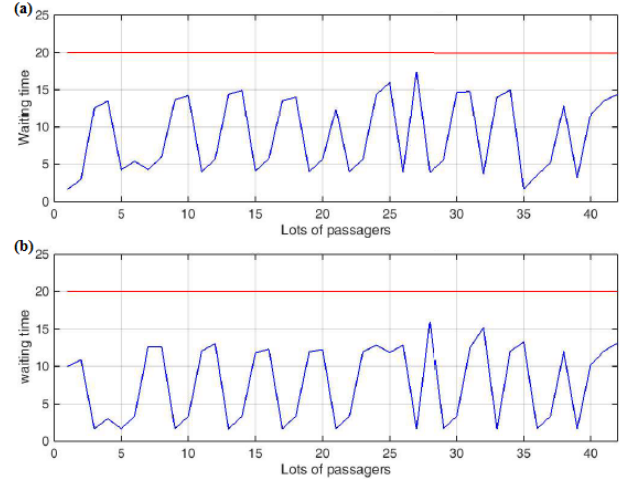

Fig. 6. Variation of the waiting time in $S_{1}$ (a) and $S_{2}$ (b) for $n=6$ buses

\section{CONCLUSION}

After describing the studied multi-modal transportation system, we have developed a CHPNs based approach for studying its performances.

In fact, we can summarize the contributions of this work as follows : (i) model the behavior of the system by a CHPN, (ii) verify and validate the proposed model by simulation, (iii) evaluate the evolution of waiting times in the two exchange stations, (iv) study the influence of the number of used buses on the passengers waiting times, and finally (v) finding minimum number of buses to use on the network to ensure the transport of all exchanged passengers while respecting a waiting time threshold.

The proposed modeling approach can be used for more other transport networks with more exchange stations. Future research will be directed toward a control approach of our MTS, study more general networks and answer a set of questions such as large scale system performances.

\section{REFERENCES}

[1] F. Chen, L. Wang, B. Jiang, and C. Wen. "A novel hybrid petri net model for urban intersection and its application in signal contro strategy" Journal of the Franklin Institute, 351(8), 4357-4380, 2014.

[2] G. Cavone, M. Dotoli, C. Seatzu, "A Survey on Petri Net Models for Freight Logistics and Transportation Systems" IEEE Transactions on Intelligent Transportation Systems, 2017.

[3] K. Outafraout, E. Chakir El Alaoui and A. Nait-Sidi-Moh. "Modeling and simulation of a Multimodal Transportation System based on Hybrid Petri Nets", the 14th IEEE International Multi-Conference on Systems, Signals and Devices (SSD-17), Marrakech-Morocco, March 28-31, p. 413-418, 2017.

[4] L. Xiaoyu, M. Zhou and A. Chiheb. "Hybrid Petri nets for modeling and analysis of microgrid systems". IEEE/CAA Journal of Automatica Sinica, vol. 3, no 4, p. 349-356, 2016.

[5] M. Bitam and H. Alla. "Performance evaluation of communication networks for distributed systems". Int. J. Computer Applications in Technology, Vol. 25, No. 4, 2006.

[6] R. David and H. Alla. "Discrete, continuous, and hybrid petri nets". Springer, 2010.

[7] R. Yamazaki and T. Nishi. "An optimization approach for congestion management for traffic signal control systems via first-order hybrid Petri nets". In Industrial Engineering and Engineering Management (IEEM), IEEE International Conference, p. 1252-1256, 2015.

[8] Y. Zhang, W. Qiang, Z. Yang, "A New Traffic Signal Control Method Based on Hybrid Colored Petri Net in Isolated Intersections". International Journal of Intelligent Transportation Systems Research, 15 (2), pp. 98-107, 2017. 